\title{
Standardized preoperative corticosteroid treatment in neonates undergoing cardiac surgery: Results from a randomized trial
}

\author{
Eric M. Graham, MD, ${ }^{\mathrm{a}}$ Andrew M. Atz, MD, ${ }^{\mathrm{a}}$ Ryan J. Butts, MD, ${ }^{\mathrm{a}}$ Nathaniel L. Baker, MS, ${ }^{\mathrm{b}}$ \\ Sinai C. Zyblewski, MD, ${ }^{a}$ Rachael L. Deardorff, MS, ${ }^{d}$ Stacia M. DeSantis, PhD, ${ }^{b}$ Scott T. Reeves, MD, ${ }^{c}$ \\ Scott M. Bradley, MD, ${ }^{\mathrm{d}}$ and Francis G. Spinale, MD, $\mathrm{PhD}^{\mathrm{d}}$
}

\begin{abstract}
Objective: A heightened inflammatory response occurs after cardiac surgery. The perioperative use of glucocorticoids has been advocated as a method to improve postoperative outcomes. Randomized prospective studies to quantify the effect of methylprednisolone on perioperative outcomes in neonatal cardiac surgery have not been performed. We sought to determine whether preoperative methylprednisolone would improve postoperative recovery in neonates requiring cardiac surgery.
\end{abstract}

\begin{abstract}
Methods: Neonates scheduled for cardiac surgery were randomly assigned to receive either 2 -dose ( 8 hours preoperatively and operatively, $\mathrm{n}=39$ ) or single-dose (operatively, $\mathrm{n}=37$ ) methylprednisolone $(30 \mathrm{mg} / \mathrm{kg}$ per dose) in a prospective double-blind trial. The primary outcome was the incidence of low cardiac output syndrome (standardized score) or death 36 hours postoperatively. Secondary outcomes were death at 30 days, interleukin-6 levels, inotropic score, fluid balance, serum creatinine, and intensive care unit and hospital stay.

Results: Preoperative plasma levels of the inflammatory cytokine interleukin-6 were reduced by 2 -fold $(P<.001)$ in the 2-dose methylprednisolone group, consistent with the anti-inflammatory effects of methylprednisolone. However, the incidence of low cardiac output syndrome was $46 \%(17 / 37)$ in the single-dose and $38 \%$ $(15 / 39)$ in the 2-dose methylprednisolone groups $(P=.51)$. Two-dose methylprednisolone was associated with a higher serum creatinine $(0.61 \pm 0.18 \mathrm{mg} / \mathrm{dL}$ vs $0.53 \pm 0.12 \mathrm{mg} / \mathrm{dL}, P=.03)$ and poorer postoperative diuresis $(-96 \pm 49 \mathrm{~mL}, P=.05)$. Inotropic requirement, duration of mechanical ventilation, intensive care unit, and hospital stay did not differ between the 2 groups.
\end{abstract}

Conclusions: Combined preoperative and intraoperative use of glucocorticoids in neonatal cardiac surgery does not favorably affect early clinical outcomes and may exacerbate perioperative renal dysfunction. (J Thorac Cardiovasc Surg 2011;142:1523-9)

The performance of corrective or palliative cardiac surgery for congenital cardiac defects can be associated with a complex postoperative course. This is particularly true when these cardiac procedures are necessary in neonates in whom an increased risk of morbidity and mortality is encountered in the early postoperative period. In adults and older pediatric patients, it has been demonstrated that one of the ubiquitous consequences of cardiopulmonary bypass (CPB) is a heightened proinflammatory state. ${ }^{1,2} \mathrm{~A}$ heightened inflammatory

\footnotetext{
From the Division of Cardiology, ${ }^{\mathrm{a}}$ Department of Pediatrics, Biostatistics and Epidemiology, ${ }^{\mathrm{b}}$ Anesthesia and Perioperative Medicine, ${ }^{\mathrm{c}}$ and Division of Cardiothoracic Surgery, ${ }^{\mathrm{d}}$ Department of Surgery, Medical University of South Carolina, Charleston, SC.

This work was supported in part by a Career Development Award from the American College of Cardiology Foundation/Pfizer Scholarship (to E.M.G.), National Institutes of Health Grants HL-057952 and HL-059165 (to F.G.S.), and the Research Service of the Department of Veterans Affairs.

Disclosures: Authors have nothing to disclose with regard to commercial support.

Received for publication Jan 20, 2011; revisions received April 1, 2011; accepted for publication April 18, 2011; available ahead of print May 23, 2011.

Address for reprints: Eric M. Graham, MD, Medical University of South Carolina, 165 Ashley Avenue MSC 915, Charleston, SC 29425 (E-mail: grahamem@ musc.edu).

0022-5223/\$36.00

Copyright (c) 2011 by The American Association for Thoracic Surgery

doi:10.1016/j.jtcvs.2011.04.019
}

state has been postulated to negatively affect cardiac function and hemodynamic status, leading to postoperative morbidity, such as low cardiac output syndrome (LCOS), shifts in fluid balance, and increased need for mechanical and pharmacologic support. ${ }^{3}$ As a result, the empirical use of prophylactic anti-inflammatory therapy, primarily through the use of glucocorticoids, has become a common practice in pediatric cardiac surgery. ${ }^{4,5}$ Outcomes data regarding the use of glucocorticoids have been variable, the dosing regimens and protocols have not been uniform, and the evaluation of preoperative steroid treatment in neonates has not been specifically examined. ${ }^{4-12}$ Accordingly, the overall goal of this study was to perform a prospective, randomized study to test the hypothesis that preoperative glucocorticoid therapy, added to standard intraoperative administration, would improve early postoperative outcomes and indices of inflammation in neonates undergoing cardiac operations requiring $\mathrm{CPB}$.

\section{MATERIALS AND METHODS \\ Rationale and Study Overview}

Although the optimal type and dose of glucocorticoid for use in the context of CPB remain to be established, the most commonly used agent is methylprednisolone (MP). ${ }^{4} \mathrm{MP}$ has been used in small pediatric cardiac 


$$
\begin{aligned}
& \text { Abbreviations and Acronyms } \\
& \begin{aligned}
\mathrm{CPB} & =\text { cardiopulmonary bypass } \\
\mathrm{ICU} & =\text { intensive care unit } \\
\mathrm{IL} & =\text { interleukin } \\
\mathrm{LCOS} & =\text { low cardiac output syndrome } \\
\mathrm{MP} & =\text { methylprednisolone } \\
\mathrm{WBC} & =\text { white blood cell }
\end{aligned}
\end{aligned}
$$

surgery series and by the Pediatric Heart Network in a randomized placebocontrolled clinical trial of Kawasaki disease treatment in children. ${ }^{6,13}$ By extrapolating from these studies, intravenous MP at $30 \mathrm{mg} / \mathrm{kg}$ of body weight was chosen for use. The timing of the preoperative dose was chosen to optimize the potential effect based on institutional experience, because pharmacokinetic data have not been reported specifically in neonates. The use of some type of intraoperative steroids during neonatal cardiac surgery is widespread. ${ }^{4,5}$ In light of the presumed beneficial effects demonstrated in both animal and small pediatric studies, and given the widespread use of MP, we considered it inappropriate to randomize neonates to a completely placebo control group. ${ }^{4,6,12}$

\section{Patient Selection, Enrollment, and Randomization}

All inpatient neonates (aged $\leq 30$ days) who were scheduled to undergo cardiac surgery involving CPB from July 2007 to July 2009 were eligible for this study (ClinicalTrials.gov Identifier: NCT00934843). Exclusion criteria included prematurity (defined as $\leq 36$ weeks gestational age) at the time of surgery, treatment with steroids in the 2 weeks before surgery, suspected infection that would contraindicate steroid use (eg, herpes), known hypersensitivity to MP or other contraindication to steroid therapy (eg, gastrointestinal bleeding), preoperative use of mechanical circulatory support or active resuscitation at the time of proposed randomization, and inability to begin the preoperative study drug at least 8 hours before surgery. The study was approved by the institutional review board, and informed written consent was obtained from the parent or legal guardian. Patients were stratified on the basis of a planned palliative or corrective procedure and then randomized by the investigational pharmacy to 1 of 2 study groups: preoperative placebo and intraoperative MP (single-dose group) or preoperative and intraoperative MP (2-dose group). All investigation and clinical site personnel were blinded to the treatment allocation until the close of the study.

\section{Perioperative Management}

Preoperative management followed the general principle of reasonable avoidance of mechanical ventilation and inotropic medications. Study medication, $30 \mathrm{mg} / \mathrm{kg}$ MP or a similar volume of normal saline, was infused over a 1-hour period 8 hours before scheduled CPB. In the operating room, general anesthesia was attained with narcotics, muscle paralysis, and inhaled anesthetic. The CPB prime included $30 \mathrm{mg} / \mathrm{kg}$ of MP in all patients. A heparin-bonded circuit was used for circulatory support. Full-flow bypass at mild hypothermia $\left(32^{\circ} \mathrm{C}\right)$ or low-flow bypass at deep hypothermia $\left(20^{\circ} \mathrm{C}-25^{\circ} \mathrm{C}\right)$ was used. Cold-blood cardioplegia was given at 20 -minute intervals during periods of aortic crossclamping. Deep hypothermic circulatory arrest was performed at $18^{\circ} \mathrm{C}$ when necessary. Acid-base management was by a $\mathrm{pH}$ stat strategy. Conventional and modified ultrafiltration were used in all cases. Leukocyte depletion filters were not used in any patient. Aprotinin was used in all patients except 1 before May 2008 and in no patient subsequently because of its unavailability. Delayed sternal closure was used in all patients undergoing a Norwood procedure and in other operations as needed. All patients were managed postoperatively in a dedicated pediatric cardiac intensive care unit (ICU). Unless significant hypotension or renal dysfunction was present, milrinone was used at a dose of $0.5 \mu \mathrm{g} / \mathrm{kg} / \mathrm{min}$.

\section{Primary and Secondary Response Variables}

The primary end point was the incidence of death or LCOS at 36 hours from admission to the ICU after surgery. The presence of LCOS was defined by the same definition used in the PRIMACORP study, ${ }^{3}$ specifically, if there were clinical signs and symptoms of low cardiac output (eg, tachycardia, oliguria, cold extremities, cardiac arrest) that required 1 or more of the following interventions: mechanical circulatory support, the escalation of existing pharmacologic circulatory support to more than $100 \%$ over baseline, or the initiation of new pharmacologic circulatory support. The determination of LCOS was made by 2 independent reviewers (EMG, AMA) and then agreed on between both reviewers before unblinding. Secondary end points included inotropic score, urine output, and total fluid balance over the first 36 hours postoperatively, and the incidence of death between 36 hours and 30 days postoperatively. The inotropic score was calculated by the equation using drug dosages in micrograms/kilograms/minute $($ dopamine + dobutamine $)+($ milrinone $\times 10)+($ epinephrine $\times 100)$ and recorded on arrival to the ICU, 4, 8, 12, 24, and 36 hours postoperatively. ${ }^{14,15}$ The highest score during this timeframe was also recorded. Secondary end points also included the duration of postoperative mechanical ventilation and ICU and hospital stay. Markers of inflammation, including white blood cell (WBC) count and the plasma level of the proinflammatory cytokine interleukin (IL)-6, were compared between groups. WBC was determined immediately preoperatively, immediately postoperatively, and at 36 hours postoperatively. Plasma samples collected before preoperative placebo/MP treatment ( 8 hours preoperatively), immediately preoperatively, on completion of modified ultrafiltration, and at 4,12 , and 24 hours postoperatively were subjected to multiplex analysis for IL-6.

\section{Sample Size Estimates}

The PRIMACORP study, from which the criteria of LCOS were derived for the present study, ${ }^{3}$ reported that milrinone reduced LCOS in neonates by approximately $16 \%(23 \%$ vs $7 \%) .{ }^{16}$ In a prior series of children undergoing cardiac surgery and $\mathrm{CPB}$, a combined preoperative and operative MP was reported to reduce the incidence of LCOS by $26 \%(40 \% \text { vs } 14 \%)^{6}$ The current study was designed with an anticipated enrollment of 87 patients per treatment arm to achieve a statistical power of $80 \%(1-\beta)$ while controlling type I error at $0.05(\alpha)$ using a more conservative estimate of LCOS rate of $33 \%$ in the single-dose MP group and an incidence of LCOS of $15 \%$ in the 2-dose MP group. After recruiting for 25 months, 76 patients had been assessed for the outcome of interest. The aggregate incidence rate of LCOS was higher than anticipated at $42 \%$ (32/76 vs an anticipated $24 \%$ ). After unblinding, the effect size was in the direction hypothesized, but this difference was only $7.5 \%$. At the time of unblinding, the conditional power to detect the hypothesized difference of $18 \%$ was calculated to determine the futility of continuing the study. ${ }^{17}$ The conditional power was determined to be $8.3 \%$ using Cytel East5 software (version 5; Cytel Inc, Cambridge, Mass). This low (conditional) probability of rejecting the null hypothesis provided adequate evidence against finding a significant effect and led to the suspension of further patient enrollment. ${ }^{18}$

\section{Data Analysis}

The continuous preoperative and postoperative clinical characteristics are presented as mean \pm standard deviation. For the categoric variables, the percent incidence is presented. Treatment group comparisons for continuous variables were performed using a Wilcoxon rank-sum test, and categoric variables were compared using a Pearson's chi-square test (Fisher exact test where appropriate). Because of the high incidence of LCOS and the prospective nature of the study, the outcome was subjected to univariate and multivariable risk regression with robust error variance 
estimates to determine unadjusted and adjusted relative risk ratios. Maximum likelihood methods were implemented to estimate the parameters using a log-linear model in SAS proc genmod using Poisson regression modeling with robust error variance estimates. ${ }^{19}$ Analysis of variance/ covariance models was used to test both unadjusted and multivariable relationships between treatment groups for blood chemistries and diuresis. A repeated-measures analysis of variance framework was used for longitudinal analysis of inotrope score, plasma creatinine, WBC, and the logarithm of IL-6. To identify potential confounding variables, a variable was considered a relevant covariate and added into the model with a $P$ value of .15 or less. Potential confounding variables included race, gender, systolic blood pressure, weight at time of surgery, and gestational age. During the execution of this study, the antifibrinolytic aprotinin was discontinued from clinical use and therefore was included in all adjusted models. Modelbased estimates are reported as the mean \pm standard error. Statistical analyses were performed with SAS (version 9.1.3; SAS Institute, Inc, Cary, $\mathrm{NC}$ ). All significance tests are 2 tailed, and no correction for multiple testing has been applied to reported $P$ values.

\section{RESULTS}

\section{Preoperative Demographics and Intraoperative Variables}

Enrollment and outcomes are shown in Figure 1. A total of 76 patients were randomly assigned to either preoperative placebo and intraoperative MP (single-dose MP group, $\mathrm{n}=37 ; 49 \%)$ or preoperative and intraoperative MP (2-dose MP group, $\mathrm{n}=39 ; 51 \%$ ) protocol. Patient demo-

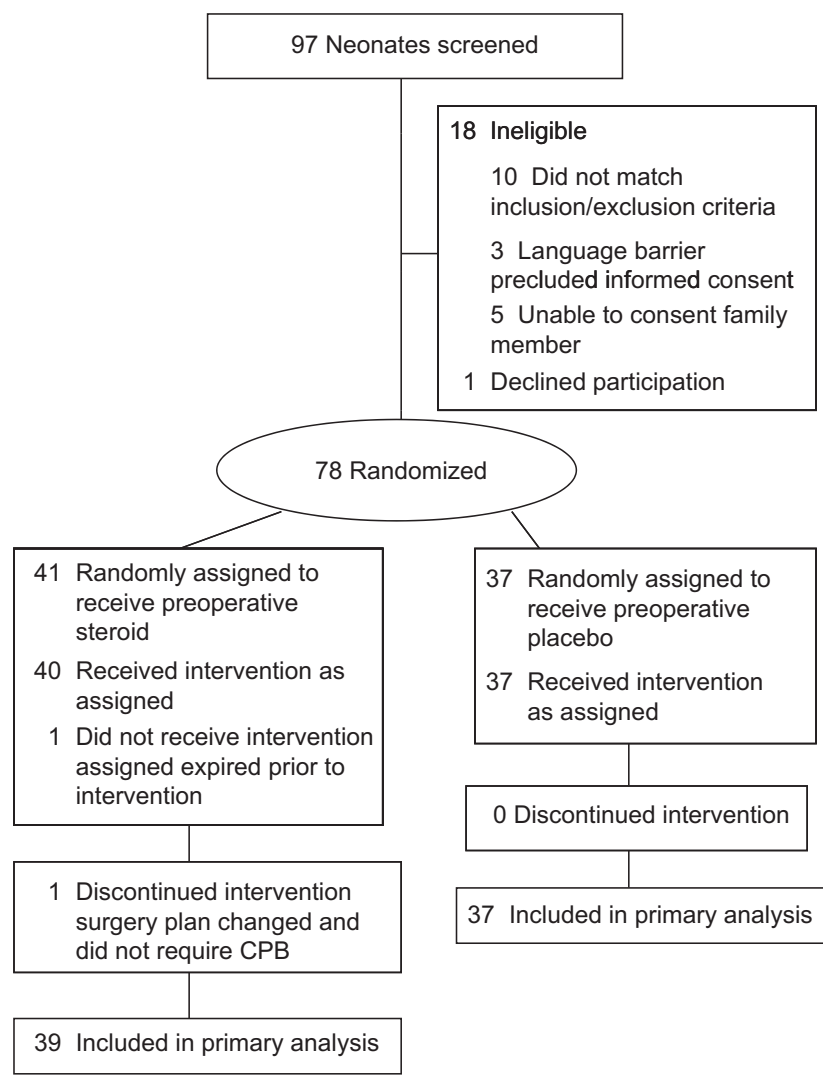

FIGURE 1. Flow of patients through the trial. $C P B$, Cardiopulmonary bypass. graphics, cardiac diagnoses, and clinical characteristics are summarized in Table 1. The groups were well balanced with respect to gender, cardiac malformation, and planned surgical procedure. The gestational age was slightly older in the 2-dose MP group, but the ages and weight at the time of surgery were similar. Intra- and perioperative parameters are shown in Table 2 . The overall CPB time, crossclamp time, use of deep hypothermic circulatory arrest, and immediate postoperative procedures were similar between the 2 MP treatment groups.

\section{Postoperative Outcomes: Primary Response Variables}

The incidence of LCOS was $46 \%$ (17/37) in the singledose MP group and 38\% (15/39) in the 2-dose MP group, with no difference between groups. By using an unadjusted univariate risk computation, the relative risk ratio for the 2-dose MP group was 0.84 (95\% confidence interval, $0.49-1.42 ; P=.51)$, indicating no reduction in the risk of LCOS with this treatment strategy. Although the treatment groups were well balanced with respect to potential confounding variables, an adjusted analysis was performed including previously mentioned covariates. The adjusted relative risk was computed for the 2-dose MP group, yielding a relative risk of 0.78 (95\% confidence interval, $0.45-1.33$; $P=.35$ ).

Overall 30-day survival was $98.7 \%$. There were no deaths in the first 36 hours postoperatively in either group. There was a single death between 36 hours and 30 days postoperatively in the single-dose MP group, secondary to gram-negative sepsis 19 days after a central shunt and pulmonary arterioplasty for tetralogy of Fallot with pulmonary atresia. Other clinical response variables, such as duration of mechanical ventilation, ICU time, and hospital stay, were similar between the 2 different MP treatment groups (Table 3).

\section{Perioperative Outcomes and Laboratory Values}

The inotrope score as a function of time postoperatively is summarized in Figure 2. Inotropic score increased in a predictable manner early postoperatively and decreased as a function of time, with no differences between groups (adjusted $\mathrm{F}_{1,70}=0.03 ; P=.86$ ). Clinical outcome variables are summarized in Table 3. The highest plasma lactate value recorded in the postoperative period was higher in the 2dose MP group (Table 3). Similarly, plasma creatinine was higher in the immediate postoperative period in the 2-dose MP group: $0.61 \pm 0.18 \mathrm{mg} / \mathrm{dL}$ compared with $0.53 \pm 0.12 \mathrm{mg} / \mathrm{dL}(P=.03$, Figure 2$)$. Total fluid administration and outputs for the first 36 hours postoperative are described in Table 3. When adjusted for fluid intake and intermittent bolus versus continuous furosemide administration, the between-group differential in fluid output $(-101$ $\pm 50 \mathrm{~mL}, P=.047)$ and urine output $(-96 \pm 49 \mathrm{~mL}$, 
TABLE 1. Preoperative demographics in neonatal patients undergoing cardiac surgery randomized tosingle-or 2-dose methylprednisolone strategy

\begin{tabular}{|c|c|c|c|}
\hline & $\begin{array}{l}\text { MP single-dose } \\
\quad \mathbf{n}=37\end{array}$ & $\begin{array}{l}\text { MP 2-dose } \\
\mathbf{n}=39\end{array}$ & $P$ value \\
\hline \multicolumn{4}{|l|}{ Age/gender } \\
\hline Gestational age at birth (wk) & $38.3 \pm 1.4$ & $38.9 \pm 1.2$ & .08 \\
\hline Gestational age at surgery (wk) & $39.5 \pm 1.4$ & $40.2 \pm 1.6$ & .04 \\
\hline Age at surgery $(\mathrm{d})$ & $8.2 \pm 5.0$ & $9.4 \pm 6.3$ & .34 \\
\hline Age $\leq 7 \mathrm{~d}, \mathrm{n}(\%)$ & $27(73 \%)$ & $23(59 \%)$ & .30 \\
\hline Weight at surgery $(\mathrm{kg})$ & $3.1 \pm 0.5$ & $3.3 \pm 0.5$ & .11 \\
\hline Prenatal diagnosis, $\mathrm{n}(\%)$ & $15(41 \%)$ & $18(46 \%)$ & .62 \\
\hline Male, $n(\%)$ & $19(51 \%)$ & $20(51 \%)$ & .99 \\
\hline Creatinine $(\mathrm{mg} / \mathrm{dL})$ & $0.51 \pm 0.15$ & $0.56 \pm 0.21$ & .25 \\
\hline Diagnosis & & & Operative procedure \\
\hline Corrective procedure & $21(57 \%)$ & $22(56 \%)$ & $P=.84$ \\
\hline Aortic arch hypoplasia with VSD & 5 & 5 & Aortic arch repair, VSD closure \\
\hline Tetralogy of Fallot & 2 & 2 & Complete repair with transannular patch \\
\hline Transposition of the great arteries \pm VSD & 11 & 10 & Arterial switch \pm VSD closure \\
\hline Truncus arteriosus & 1 & 3 & Complete repair \\
\hline Other biventricular repair & 2 & 2 & \\
\hline Palliative procedure & $16(43 \%)$ & $17(44 \%)$ & $P=.84$ \\
\hline Hypoplastic left heart syndrome & 6 & 8 & Norwood procedure \\
\hline Other single ventricle lesions & 8 & 7 & $\begin{array}{l}\text { Norwood, mBTS, RV-PA shunt, pulmonary } \\
\text { artery operation }\end{array}$ \\
\hline Pulmonary atresia with intact ventricular septum & 0 & 2 & RVOT patch, mBTS \\
\hline Tetralogy of Fallot with pulmonary atresia & 2 & 0 & mBTS and pulmonary arterioplasty \\
\hline
\end{tabular}

$V S D$, Ventricular septal defect; $m B T S$, modified Blalock-Taussig shunt; $R V-P A$, right ventricle to pulmonary artery; $M P$, methylprednisolone; $R V O T$, right ventricular outflow tract. Data are reported as mean \pm standard deviation. Data are reported as number (\%). Single-dose: $30 \mathrm{mg} / \mathrm{kg}$ MP in CPB prime; 2 -dose: $30 \mathrm{mg} / \mathrm{kg}$ preoperative and 30 $\mathrm{mg} / \mathrm{kg}$ MP in CPB prime.

$P=.052)$ was slightly worse in the 2-dose MP group. IL-6 values before randomization and MP treatment were equivalent between groups (Figure 2), but were lower preoperatively in the 2-dose MP group (adjusted $\mathrm{F}_{1,65}=45.0$; $P<.001$ ), consistent with the anti-inflammatory effects of this pharmacologic protocol. Plasma IL-6 increased equivalently in both groups by approximately 2 -fold in the postoperative period (steroid effect: $\mathrm{F}_{1,65}=0.10 ; P=.909$ ) regardless of group assignment and consistent with past re-

TABLE 2. Intraoperative and perioperative parameters in neonatal patients undergoing cardiac surgery randomized to single- or 2-dose methylprednisolone strategy

\begin{tabular}{lccc}
\hline & $\begin{array}{c}\text { MP single-dose } \\
\mathbf{n = 3 7}\end{array}$ & $\begin{array}{c}\text { MP 2-dose } \\
\mathbf{n}=\mathbf{3 9}\end{array}$ & $\begin{array}{c}\boldsymbol{P} \\
\text { value }\end{array}$ \\
\hline CPB time (min) & $168 \pm 71$ & $156 \pm 39$ & .62 \\
Aortic crossclamp time (min) & $71 \pm 36$ & $67 \pm 28$ & .83 \\
Use of DHCA, n (\%) & $14(38 \%)$ & $17(44 \%)$ & .61 \\
DHCA time (min) & $23 \pm 14$ & $21 \pm 10$ & .64 \\
Modified ultrafiltration time (min) & $12 \pm 2$ & $13 \pm 3$ & .13 \\
Aprotinin administration, n (\%) & $18(49 \%)$ & $16(41 \%)$ & .50 \\
Delayed sternal closure, $\mathrm{n}(\%)$ & $20(54 \%)$ & $18(46 \%)$ & .49 \\
\hline
\end{tabular}

$M P$, Methylprednisolone; $C P B$, cardiopulmonary bypass; $D H C A$, deep hypothermic circulatory arrest. Data are reported as mean \pm standard deviation. Single-dose: 30 $\mathrm{mg} / \mathrm{kg}$ MP in CPB prime; 2-dose: $30 \mathrm{mg} / \mathrm{kg}$ preoperative and $30 \mathrm{mg} / \mathrm{kg}$ MP in CPB prime. ports. ${ }^{6,7,20}$ The WBC count was similar between groups in the preoperative period $\left(11.9 \pm 0.5 \times 10^{-3} / \mathrm{mm}^{3}\right.$ in the single-dose MP group vs $10.7 \pm 0.6 \times 10^{-3} / \mathrm{mm}^{3}$ in the 2 -dose MP group, $P=.14$ ). However, the WBC count in the immediate postoperative period was significantly higher in the 2-dose MP group $\left(7.8 \pm 0.5 \times 10^{-3} / \mathrm{mm}^{3}\right.$ in the single-dose MP group vs $9.5 \pm 0.6 \times 10^{-3} / \mathrm{mm}^{3}$ in the 2 dose MP group, $P=.04$ ). At later postoperative periods, the WBC count was similar between groups.

\section{DISCUSSION}

The fundamental premise of this study was that if glucocorticoids impart a protective effect in the context of neonatal cardiac surgery, then it would be expected that the 2-dose MP protocol would translate into improved early postoperative outcomes. The prospective randomization yielded a balanced set of patients in each treatment arm and thereby provided control for a number of potentially confounding variables. The unique findings from the present study are 3 -fold. First, the absolute incidence and relative risk for LCOS were not different between the MP dosing strategies. Second, plasma creatinine, lactate level, and fluid balance were actually worse in the 2-dose MP group, with no differences in other postoperative outcome variables between the MP dosing strategies. Third, although the 2-dose MP 
TABLE 3. Univariate postoperative outcomes in neonatal patients undergoing cardiac surgery randomized to single- or 2-dose methylprednisolone strategy

\begin{tabular}{|c|c|c|c|}
\hline & $\begin{array}{c}\text { MP } \\
\text { single-dose } \\
\mathbf{n}=\mathbf{3 7} \\
\end{array}$ & $\begin{array}{c}\text { MP } \\
\text { 2-dose } \\
n=39 \\
\end{array}$ & $\begin{array}{c}P \\
\text { value } \\
\end{array}$ \\
\hline $\begin{array}{l}\text { Mechanical cardiac support, } \\
\text { n (\%) }\end{array}$ & $3(8 \%)$ & $1(3 \%)$ & .35 \\
\hline Mechanical ventilation (d) & $5.8 \pm 7.9$ & $6.8 \pm 9.5$ & .21 \\
\hline ICU stay $(\mathrm{d})$ & $11.0 \pm 18.5$ & $10.8 \pm 12.8$ & .19 \\
\hline Hospital stay (d) & $23 \pm 25$ & $22 \pm 15$ & .34 \\
\hline Total fluid in at $36 \mathrm{~h}(\mathrm{~mL})$ & $575 \pm 145$ & $586 \pm 156$ & .77 \\
\hline Total fluid out at $36 \mathrm{~h}(\mathrm{~mL})$ & $600 \pm 250$ & $558 \pm 203$ & .43 \\
\hline $\begin{array}{l}\text { Total urine output at } \\
36 \mathrm{~h}(\mathrm{~mL})\end{array}$ & $498 \pm 227$ & $453 \pm 213$ & .38 \\
\hline C-reactive protein at $36 \mathrm{~h}$ & $6.2 \pm 3.6$ & $6.3 \pm 4.9$ & .89 \\
\hline Highest inotropic score & $14.4 \pm 3.6$ & $15.0 \pm 3.9$ & .43 \\
\hline Highest lactate (mmol/L) & $3.8 \pm 2.4$ & $5.2 \pm 3.5$ & .05 \\
\hline $\begin{array}{l}\text { Highest temperature in first } \\
36 \mathrm{~h}\left({ }^{\circ} \mathrm{C}\right)\end{array}$ & $37.5 \pm 0.3$ & $37.4 \pm 0.4$ & .70 \\
\hline Highest glucose & $145 \pm 27$ & $167 \pm 54$ & .12 \\
\hline Insulin drip, $\mathrm{n}(\%)$ & 0 & $3(8 \%)$ & .24 \\
\hline Infection, $\mathrm{n}(\%)$ & $5(14 \%)$ & $5(13 \%)$ & .96 \\
\hline Poor wound healing, $\mathrm{n}(\%)$ & $1(3 \%)$ & $5(13 \%)$ & .20 \\
\hline $\begin{array}{l}\text { Additional postoperative } \\
\text { steroids, } \mathrm{n}(\%)\end{array}$ & $6(16 \%)$ & $8(21 \%)$ & .85 \\
\hline $\begin{array}{l}\text { Thyroid supplementation, } \\
\mathrm{n}(\%)\end{array}$ & $8(22 \%)$ & $11(28 \%)$ & .51 \\
\hline
\end{tabular}

protocol reduced preoperative cytokine levels, consistent with an anti-inflammatory effect, this effect did not persist into the postoperative period.

There is widespread use of perioperative glucocorticoid administration in pediatric cardiac surgery, with $80 \%$ to $97 \%$ of centers using some form of glucocorticoid therapy. ${ }^{4,5}$ However, the type of glucocorticoid and the dosing and timing of administration are highly variable, reinforcing the lack of convincing data to guide therapeutic administration. In the largest pediatric study, Pasquali and colleagues ${ }^{21}$ used the Pediatric Health Information Systems Database to evaluate the outcomes of corticosteroids in children aged 0 to 18 years undergoing congenital heart operations. Data from 46,730 patients were included, $54 \%$ of whom received corticosteroids. Multivariable analysis found no difference in postoperative mortality between corticosteroid recipients and nonrecipients. Corticosteroids were associated with longer length of stay, greater infection, and greater use of insulin. There was no difference in duration of mechanical ventilation, similar to the present study. In a subanalysis of 10,018 neonates, corticosteroids were associated with significantly longer postoperative ICU length of stay and greater use of insulin. Although Pasquali and colleagues' study has the in- herent limitations of any observational study using a large database, and the authors were unable to evaluate the impact of different dosing regimens or exact timing or corticosteroid administration in relation to surgery, it is in agreement with the current prospective randomized study.

Few controlled trials of glucocorticoids in the setting of CPB in pediatrics can be found. Bronicki and colleagues ${ }^{7}$ randomized 29 children (mean age of 28 months) to receive dexamethasone $(1 \mathrm{mg} / \mathrm{kg}, \mathrm{n}=15)$ or placebo $(\mathrm{n}=14) 1$ hour before $\mathrm{CPB}$. Both groups demonstrated a significant elevation in IL-6 after CPB, similar to our findings. However, dexamethasone-treated patients had a blunted IL-6 response when compared with placebo-treated patients. This corresponded to an improvement in renal function (defined as the number of patients whose postoperative creatinine level increased $\geq 0.2 \mathrm{mg} / \mathrm{dL}$ above baseline) and respiratory function, resulting in shorter durations of mechanical ventilation and ICU stay in the treatment group. Despite blinded randomization, there may have been more complex diagnoses and operations in the placebo group. The improvement in renal function is in contrast with our findings of an elevated postoperative creatinine and worse diuresis and fluid balance in the 2-dose MP group. Although MP is a potent glucocorticoid, it does have some mineralocorticoid activity. The high doses used in the current study may explain the decreased urine output and worse fluid balance in patients receiving 2-dose MP.

Lindberg and colleagues ${ }^{9}$ randomized 40 patients weighing more than $10 \mathrm{~kg}$ (mean age of 52 months) to receive dexamethasone $(1 \mathrm{mg} / \mathrm{kg}, \mathrm{n}=20)$ or placebo $(\mathrm{n}=20)$ after the induction of anesthesia. C-reactive protein, as a marker of inflammation, was lower in the treatment group on the first postoperative day. Contrary to the findings of Bronicki and colleagues, ${ }^{7}$ there were no significant differences in clinical outcomes between treatment groups.

Schroeder and associates ${ }^{6}$ randomized 29 children (mean age 4 months $)$ to MP $(30 \mathrm{mg} / \mathrm{kg}, \mathrm{n}=15)$ or placebo $(\mathrm{n}=14)$ 4 hours before CPB. As in our study, MP (30 mg/kg) was administered in the pump prime in all patients. Serum IL6 was lower immediately and 4 hours after CPB in the combined MP compared with the intraoperative only group, but equally elevated in both groups by 24 hours. A lower core body temperature, reduced fluid requirements, and improved oxygen delivery were found in the combined therapy group. Trends toward shorter ICU stay $(P=.07)$ and incidence of $\operatorname{LCOS}(P=.11)$ were found in the combined treatment group. However, similar to our findings, there were no differences in duration of mechanical ventilation or inotropic requirements between groups.

Consistent with the findings of the present study, a recent meta-analysis (including the previously mentioned studies) failed to demonstrate any benefit in clinical outcomes with glucocorticoid administration. ${ }^{10}$ Only 2 of the studies included any neonates. Unique to the current study is the 

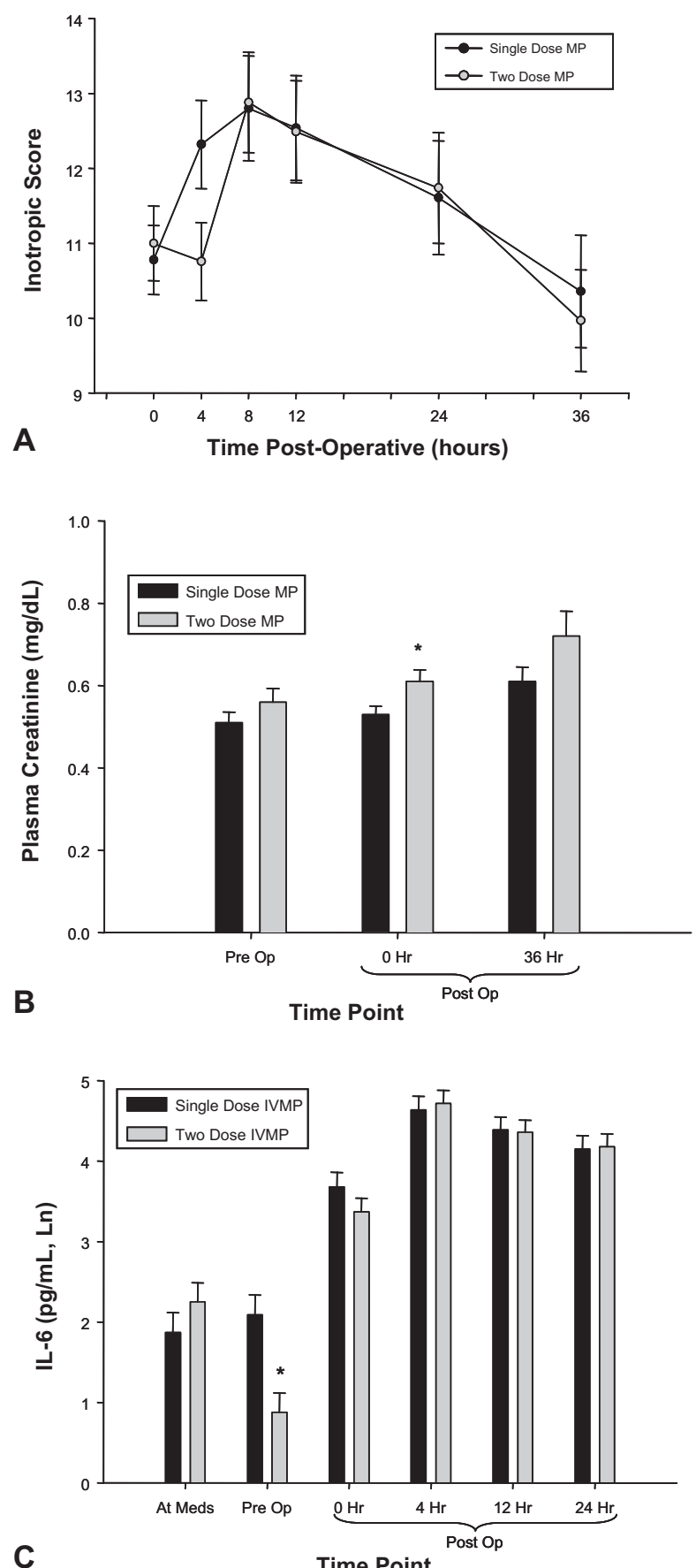

FIGURE 2. Perioperative outcomes. A, Inotrope score was computed postoperatively in both the single- and 2-dose MP groups. There was an expected increase in inotrope score with respect to time $\left(\mathrm{F}_{5,70}=12.3\right.$, $P<.001)$. Although there was a slight reduction in inotrope score in the 2-dose MP group at 4 hours postoperatively, this did not reach statistical significance $(P=.08)$. At later postoperative time points, inotrope score was virtually equivalent between groups. B, Plasma creatinine values obtained preoperatively, immediately postoperatively, and at 36 hours postoperatively for the single- and 2-dose MP groups. Plasma creatinine was significantly higher in the 2-dose MP group immediately postoperatively $\left({ }^{*} P=.03\right)$. C, The proinflammatory cytokine IL-6 was measured before sole inclusion of neonates, resulting in the inclusion of complex corrective and palliative operations and the potential increased morbidity associated with them. By combining the other studies, there were only 7 of 128 patients who underwent a palliative procedure (either complete or superior cavopulmonary connection). Considered in total, the past studies and the present study call into question the overall utility of perioperative steroids in pediatric cardiac surgery.

\section{Study Limitations}

Limitations of the current study include the lack of a true placebo group. Thus, recommendations for or against intraoperative MP cannot be made. The widespread use of MP and our decision to exclude a placebo arm were further confirmed in the recently published trial comparing shunt types used in Norwood surgery. ${ }^{22}$ Of the 549 patients undergoing the surgery, 498 (91\%) received some type of steroid during the surgery (Pediatric Heart Network, unpublished data, 2010). Although this study was more than $90 \%$ powered to detected a difference in the primary outcome of LCOS, it may have been inadequately powered to detected subtle differences in other clinical outcomes. This, however, seems unlikely given the almost identical important clinical outcomes, such as inotropic score, duration of mechanical ventilation, ICU, and hospital stay. The results of our trial do not preclude the efficacy of other glucocorticoid regimens.

\section{CONCLUSIONS}

In the largest pediatric and only exclusively neonatal randomized trial comparing the efficacy of preoperative glucocorticoid therapy with intraoperative glucocorticoid therapy alone for neonates undergoing corrective and palliative cardiac operations requiring $\mathrm{CPB}$, our data do not provide support for the addition of a preoperative dose of MP to a standard intraoperative dose alone. Although preoperative dosing was associated with an improvement in preoperative inflammatory markers, this did not correlate with improved clinical outcomes. To the contrary, preoperative MP dosing was associated with a higher immediate postoperative serum creatinine level and worse diuresis and fluid balance over the first 36 hours postoperatively. In light of these findings, the routine use of preoperative glucocorticoids in

randomization to the MP protocol (pre-MP), immediately preoperatively, immediately following modified ultrafiltration, and at 4, 12, and 24 hours postoperatively. The 2-dose MP group, in which MP was administered preoperatively and intraoperatively, resulted in a significant reduction in IL-6 values in the immediate preoperative period, consistent with an antiinflammatory effect $(* P<.001)$. In the postoperative period, plasma IL-6 values significantly increased $\left(\mathrm{F}_{3,66}=34.7, P<.001\right)$, with no difference between the single- and 2-dose MP groups. All data are presented as mean \pm standard error of the mean. $M P$, Methylprednisolone; IVMP, intravenous methylprednisolone; $I L-6$, interleukin-6. 
neonatal cardiac surgery needs to be reconsidered. A randomized trial comparing intraoperative glucocorticoid to placebo is indicated.

\section{References}

1. Tarnok A, Emmrich F. Immune consequences of pediatric and adult cardiovascular surgery: report of the 7th Leipzig workshop. Cytometry B Clin Cytom. 2003; 54:54-7.

2. Duval EL, Kavelaars A, Veenhuizen L, van Vught AJ, van de Wal HJ, Heijnen CJ. Pro- and anti-inflammatory cytokine patterns during and after cardiac surgery in young children. Eur J Pediatr. 1999;158:387-93.

3. Hoffman TM, Wernovsky G, Atz AM, Kulik TJ, Nelson DP, Chang AC, et al. Efficacy and safety of milrinone in preventing low cardiac output syndrome in infants and children after corrective surgery for congenital heart disease. Circulation. 2003;107:996-1002.

4. Checchia PA, Bronicki RA, Costello JM, Nelson DP. Steroid use before pediatric cardiac operations using cardiopulmonary bypass: an international survey of 36 centers. Pediatr Crit Care Med. 2005;6:441-4.

5. Allen M, Sundararajan S, Pathan N, Burmester M, Macrae D. Anti-inflammatory modalities: their current use in pediatric cardiac surgery in the United Kingdom and Ireland. Pediatr Crit Care Med. 2009;10:341-5.

6. Schroeder VA, Pearl JM, Schwartz SM, Shanley TP, Manning PB, Nelson DP. Combined steroid treatment for congenital heart surgery improves oxygen delivery and reduces postbypass inflammatory mediator expression. Circulation. 2003; 107:2823-8

7. Bronicki RA, Backer CL, Baden HP, Mavroudis C, Crawford SE, Green TP. Dexamethasone reduces the inflammatory response to cardiopulmonary bypass in children. Ann Thorac Surg. 2000;69:1490-5.

8. Varan B, Tokel K, Mercan S, Donmez A, Aslamaci S. Systemic inflammatory response related to cardiopulmonary bypass and its modification by methyl prednisolone: high dose versus low dose. Pediatr Cardiol. 2002;23:437-41.

9. Lindberg L, Forsell C, Jogi P, Olsson AK. Effects of dexamethasone on clinical course, C-reactive protein, S100B protein and von Willebrand factor antigen after paediatric cardiac surgery. Br J Anaesth. 2003;90:728-32.

10. Robertson-Malt S, Afrane B, El BM. Prophylactic steroids for pediatric open heart surgery. Cochrane Database Syst Rev. 2007;4:CD005550.
11. Gessler P, Hohl V, Carrel T, Pfenninger J, Schmid ER, Baenziger O, et al. Administration of steroids in pediatric cardiac surgery: impact on clinical outcome and systemic inflammatory response. Pediatr Cardiol. 2005;26:595-600.

12. Lodge AJ, Chai PJ, Daggett CW, Ungerleider RM, Jaggers J. Methylprednisolone reduces the inflammatory response to cardiopulmonary bypass in neonatal piglets: timing of dose is important. J Thorac Cardiovasc Surg. 1999;117: 515-22.

13. Newburger JW, Sleeper LA, McCrindle BW, Minich LL, Gersony W, Vetter VL, et al. Randomized trial of pulsed corticosteroid therapy for primary treatment of Kawasaki disease. N Engl J Med. 2007;356:663-75.

14. Wernovsky G, Wypij D, Jonas RA, Mayer JE Jr, Hanley FL, Hickey PR, et al. Postoperative course and hemodynamic profile after the arterial switch operation in neonates and infants. A comparison of low-flow cardiopulmonary bypass and circulatory arrest. Circulation. 1995;92:2226-35.

15. Gaies MG, Gurney JG, Yen AH, Napoli ML, Gajarski RJ, Ohye RG, et al. Vasoactive-inotropic score as a predictor of morbidity and mortality in infants after cardiopulmonary bypass. Pediatr Crit Care Med. 2010;11:234-8.

16. Hoffman TM, Wessel DL, Chang AC, Kulik TL, Atz AM, Nelson DP, et al. Milrinone usage in the neonate following congenital cardiac surgery: experience from the PRIMACORP Trial. Society for Pediatric Research E-PAS. 2007:617360.6 (abstr). Available at: http://www.pas-meeting.org/2011Denver/ abstract_archives.asp.

17. Halperin M, Lan KK, Ware JH, Johnson NJ, DeMets DL. An aid to data monitoring in long-term clinical trials. Control Clin Trials. 1982;3:311-23.

18. Lachin JM. A review of methods for futility stopping based on conditional power. Stat Med. 2005;24:2747-64.

19. Zou G. A modified Poisson regression approach to prospective studies with binary data. Am J Epidemiol. 2004;159:702-6.

20. Tarnok A, Schneider P. Pediatric cardiac surgery with cardiopulmonary bypass: pathways contributing to transient systemic immune suppression. Shock. 2001; 16(Suppl 1):24-32.

21. Pasquali SK, Hall M, Li JS, Peterson ED, Jaggers J, Lodge AJ, et al. Corticosteroids and outcome in children undergoing congenital heart surgery: analysis of the Pediatric Health Information Systems database. Circulation. 2010;122: 2123-30.

22. Ohye RG, Sleeper LA, Mahony L, Newburger JW, Pearson GD, Lu M, et al Comparison of shunt types in the Norwood procedure for single-ventricle lesions. N Engl J Med. 2010;362:1980-92. 